\title{
Multiuser Delay-Tracking CDMA Receiver
}

\author{
Wei Zha \\ Department of Electrical and Computer Engineering, Faculty of Applied Science, Queen's University, \\ Kingston, Ontario, Canada K7L 3N6 \\ Email:wzha@ee.queensu.ca
}

\section{Steven D. Blostein}

Department of Electrical and Computer Engineering, Faculty of Applied Science, Queen's University, Kingston, Ontario, Canada K7L 3N6

Email:sdb@ee.queensu.ca

Received 31 January 2002 and in revised form 7 May 2002

\begin{abstract}
We propose to integrate delay tracking into a successive interference cancellation (SIC) code division multiple access (CDMA) multiuser detector. Delay-robust SIC was initially proposed for CDMA multiuser detection when there exist time delay estimation errors. When the delay error is small, the true user signature vector can be approximately expanded around the estimated time delay as a linear combination of an estimated signature vector and an error vector. The amplitude of the error signal can be recursively estimated and cancelled in the multistage SIC iterations by using the tentative feedback decisions. Since the relative amplitude of the error signal provides information about the delay error, we propose the application of error signal feedback to improve the delay estimation. In a sliding window implementation, the delay-robust SIC is used to track multiple time varying user delays. For constant or slowly varying channels, a soft-decision function can be used to improve the delay-robust SIC. For fast fading channels, a linear-decision function is used. Delay-tracking results for both rectangular chip pulses and band-limited chip pulses are shown.
\end{abstract}

Keywords and phrases: CDMA, multiuser detection, synchronization, delay tracking, successive interference cancellation.

\section{INTRODUCTION}

The capacity of a code division multiple access (CDMA) system is limited by the multiple access interference (MAI) from other users. CDMA multiuser detection at the basestation, which utilizes known user spreading codes for detection, is an effective method to suppress MAI and improve receiver performance. The optimal multiuser detector has exponential computational complexity [1]. Several low complexity multiuser detectors including the decorrelating detector, MMSE detector, successive interference cancellation (SIC) [2], and parallel interference cancellation (PIC) receiver have been proposed [3].

These multiuser detectors all need accurate time delay information from all users. If the time delays are estimated at the receiver even within a small error, the performance will degrade dramatically [4]. There is a rich literature on initial delay estimation (acquisition) of either a single user or of all users $[5,6]$. However, little research has addressed the problem of tracking the multiuser delays after the initial acquisition.

Known multiuser delay-tracking receivers can be categorized into two types: those based on the extended Kalman filter (EKF) $[7,8,9]$ and those based on delay-locked loops
(DLLs) combined with interference cancellation [10, 11]. Both types of delay trackers require the initial conditions (time delay and amplitude) to be close to their true values or they will not converge.

The EKF-based delay tracker of $[7,8]$ can track both complex channel gain and time delay variations. However, $[7,8]$ only demonstrate tracking ability for two users. The largest number of users that can track simultaneously is not investigated and is not known $[7,8]$. The minimum mean square error EKF (MMSE-EKF) delay tracker of [9] uses $K$ disjoint (separate) EKFs, one for each of the $K$ users, so its complexity is lower than the EKF trackers of $[7,8]$ which track the $K$ users' parameters jointly. The MAI is modeled as colored Gaussian noise and suppressed by the whitening filter in [9]. Its performance is close to that of the ideal MMSE detector, and [9] also demonstrated tracking capability for a large number of users. However, [9] proposes a one-shot detector which is inherently suboptimal [12]. The EKF-based delay tracker also has high computational complexity since an innovation matrix inverse update is required for every symbol.

The parallel interference cancellation (PIC) based delay tracker combines the PIC with DLL $[10,11]$. This approach is based on a finite memory length approximation of the 
infinite memory length detector, that is, neglecting edge effects [13]. The PIC can track a large number of users under ideal power control conditions, that is, a near-far ratio of $0 \mathrm{~dB}$. However, the DLL is not fully integrated into the PIC iterations. When the near-far ratio increases to $10 \mathrm{~dB}$, it breaks down since the residual interference, due to delay errors from the strong users, causes the weak users to make incorrect symbol decisions.

The objective in this paper is to improve the above approaches by using a delay-robust SIC for delay tracking. The delay-robust SIC is based on a Taylor expansion of the true user signature vector into the sum of an estimated signature vector and an error vector. The early-late DLL is widely used in single-user receivers for tracking time delay variations [14]. Since the error vector used in the proposed delayrobust SIC is equivalent to a single-branch implementation of the early-late DLL, the delay-robust SIC implicitly combines a DLL into the SIC. Due to its robustness to delay errors even under severe near-far conditions, the tracking ability of the weakest users can be greatly improved.

The SIC regenerates and cancels the interference from other users before data detection of the desired user. The delay-robust SIC adds a delay error signal estimation and cancellation procedure onto the standard multistage SIC algorithm by using tentative decision-feedback. Since the amplitude of the delay error signal is proportional to the time delay error, the time delay error estimate is implicitly obtained by the delay-robust SIC and can be used to refine the delay estimate. To track the users' time delays, the delayrobust SIC is implemented as a sliding window. The delay error estimate of the current window is used to update the time delay information used in the next window.

If the channel is constant or varies slowly, the channel can be accurately estimated. A soft-decision function can be used in the delay-robust SIC to deliver close to single-user bit error rates (BER). For fast fading channels, we may use a linear-decision function combined with a delay-robust SIC to achieve robustness to both fading and time delay error.

Related research appears recently in $[15,16]$. However, [15] assumes a fixed global timing error identical for all users and rectangular pulses. The method in [15] does not utilize the error vector combined with decision-feedback to estimate different users' random timing error. In [15], the performance is evaluated under ideal power control conditions. Here, we consider drifting timing errors that differ among users and arbitrary band-limited pulses. We evaluated the performance under severe near-far conditions. Although the local reference adjustment [15, Scheme 3] appears to be somewhat similar to our proposed method in updating the user signature sequence according to the estimated timing error, this updating is not used in the random timing error estimation procedure. Thus, the two algorithms are quite different and [15] cannot be generalized to the proposed method in this paper. In [16], it is assumed that the data is known, while we consider that the data symbols are unknown and to be estimated iteratively in the timing error estimation procedure. Although [16] can also be used in a decision-directed fashion, we emphasize that data detection and timing error estimation must be performed jointly for best performance.

Section 2 describes the system model. Section 3 introduces the delay-robust SIC and applies it to the multiuser delay tracking. Section 4 provides simulation results for both constant and multipath fast fading CDMA channels. Section 4.4 considers the delay tracking for multipath fast fading CDMA channels. Section 5 discusses the problem with delay tracking in multipath fading multiuser CDMA systems and the possible future directions.

\section{SYSTEM MODEL}

We consider the base station receiver for the asynchronous uplink multipath fading CDMA channel with binary phase shift keying (BPSK) modulation.

The equivalent baseband received signal at the basestation is

$$
r(t)=\sum_{i=-\infty}^{\infty} \sum_{k=1}^{K} \sum_{l=1}^{L} \alpha_{k, l}(i) b_{k}(i) \tilde{s}_{k}\left(t-i T-\tau_{k, l}\right)+n(t),
$$

where $b_{k}(i) \in\{+1,-1\}$ is the $k$ th user's data symbol for the $i$ th time interval, $\tilde{s}_{k}(t)$ is the $k$ th user's normalized signature waveform, $n(t)$ is the white Gaussian noise, $\tau_{k, l} \in[0, T)$ is the $k$ th user's propagation delay for the $l$ th path, $T$ is the symbol duration, and $K$ is the total number of users. The amplitude is absorbed into the complex channel gain for the $l$ th path of the $k$ th user, $\alpha_{k, l}(i)$. The channel is assumed to remain constant for the duration of a symbol interval and varies from symbol to symbol. For simplicity, the number of multipaths, $L$, is assumed to be the same for all users.

In (1), the normalized signature waveform of user $k$, $\tilde{s}_{k}(t)$, is

$$
\tilde{s}_{k}(t)=\sum_{j=0}^{N-1} c_{k}(j) h\left(t-j T_{c}\right),
$$

where $N=T / T_{c}$ is the spreading factor, $\left\{c_{k}(j)\right\}_{j=0}^{N-1}$ is the spreading code, $T_{c}$ is the chip duration, and $h(t)$ is the chip pulse. The spreading codes of all users are assumed to be known at the receiver.

We use an observation window of length $(M+1) T$ to detect the $M$ transmitted data symbols from each user, $b_{k}(i)$, $i=1, \ldots, M$ and $k=1, \ldots, K$. Due to asynchronism $\tau_{k, l} \in$ $[0, T)$, we note that the observation interval must be of length $(M+1) T$ seconds.

After chip-matched filtering and chip-rate sampling, the received signal is discretized into a vector of $(M+1)$ observations,

$$
\mathbf{r}=\sum_{i=1}^{M} \sum_{k=1}^{K} \sum_{l=1}^{L} \alpha_{k, l}(i) b_{k}(i) \mathbf{d}_{k, l}(i)+\mathbf{n},
$$

where $\mathbf{d}_{k, l}(i)$ is the discretized signature waveform of user $k$ for the $i$ th symbol and $l$ th path and $\mathbf{n}$ is the noise vector. The received vector $\mathbf{r}$ is the concatenation of $(M+1)$ vectors, each 


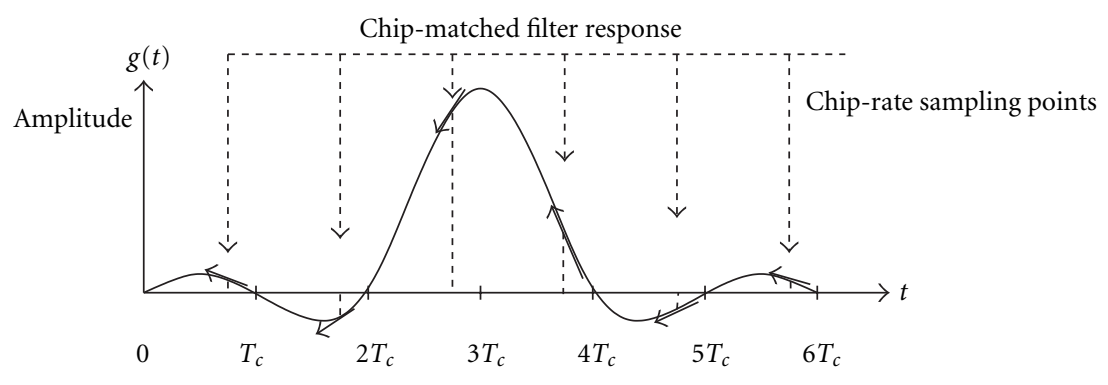

FIGURE 1: Sampling of the chip-matched filter response for truncated band-limited chip-pulse shapes. The solid arrows represent the firstorder derivative of the chip-matched filter response at the sampling points with estimated time delay.

of length $N$, that is,

$$
\mathbf{r}=\left[\mathbf{r}^{T}(1) \mathbf{r}^{T}(2) \cdots \mathbf{r}^{T}(M+1)\right]^{T} \in \mathscr{C}^{(M+1) N},
$$

where the element vector $\mathbf{r}(m)$ in (4) corresponds to the $m$ th observation interval $[(m-1) T, m T]$

$$
\mathbf{r}(m)=[r(m N+1) \cdots r(m N+N)]^{T} \in \mathscr{C}^{N} .
$$

Similarly, we may organize the zero-mean white Gaussian noise vector as

$$
\mathbf{n}=\left[\mathbf{n}^{T}(1) \mathbf{n}^{T}(2) \cdots \mathbf{n}^{T}(M+1)\right]^{T} \in \mathscr{C}^{(M+1) N} .
$$

The time delay of $l$ th path of the $k$ th user is decomposed into an integer, $p_{k, l}$, and a fractional part, $\delta_{k, l}$, where $\tau_{k, l}=$ $\left(p_{k, l}+\delta_{k, l}\right) T_{c}, p_{k, l} \in\{0,1, \ldots, N-1\}$, and $\delta_{k, l} \in[0,1)$.

We denote $\mathbf{c}_{k}\left(p_{k, l}, i\right)$ as $\mathbf{c}_{k}$ right-shifted by $(i-1) N+p_{k, l}$ chips, where $c_{k} \in \mathscr{R}^{(M+1) N}$ is the $k$ th user's spreading code vector for the $(M+1) T$ length interval defined as

$$
\mathbf{c}_{k}=[c_{k}(0) c_{k}(1) \cdots c_{k}(N-1) \underbrace{00 \cdots 0}_{M N}]^{T} .
$$

For the general band-limited chip pulse shapes $h(t)$, the received discretized signature waveform of the $i$ th symbol of the $l$ th path of the $k$ th user, $\mathbf{d}_{k, l}(i)$, is the convolution of the user spreading codes with the chip-matched filter response at the sampling points, that is,

$$
\mathbf{d}_{k, l}(i)=\mathbf{c}_{k}\left(p_{k, l}, i\right) * \mathbf{g}_{k, l}
$$

where vector $\mathbf{g}_{k, l}$ is the $l$ th path of the $k$ th user's chip-matched filter response at the chip-rate sampling points, as shown in Figure 1. If the chip-matched filter response is truncated to length $P T_{c}$, then the vector $\mathbf{g}_{k, l}$ will be of length $P$, and the signature waveform $\mathbf{d}_{k, l}(i)$ will have $N+P-1$ nonzero elements. The $m$ th element of $\mathbf{g}_{k, l}$ is given by

$$
\begin{gathered}
g_{k, l}(m)=\int_{-\infty}^{\infty} h\left(\tau-\delta_{k, l} T_{c}\right) h^{*}\left(m T_{c}-\tau\right) d \tau \\
m \in\{1,2, \ldots, P\}
\end{gathered}
$$

For the special case of rectangular chip pulse $h(t)$ with duration $\left[0, T_{c}\right), P=2$ and $\mathbf{g}_{k, l}=\left[\left(1-\delta_{k, l}\right) \delta_{k, l}\right]$. Substitut- ing into (8), the discretized signature waveform is

$$
\mathbf{d}_{k, l}(i)=\left(1-\delta_{k, l}\right) \mathbf{c}_{k}\left(p_{k, l}, i\right)+\delta_{k, l} \mathbf{c}_{k}\left(p_{k, l}+1, i\right) .
$$

The received signal vectors $\mathbf{r}(i)$ over the $(M+1)$ observations, $i=1, \ldots, M+1$, provide sufficient statistics for detecting the transmitted data symbols from the $K$ users.

\section{DELAY-ROBUST SIC WITH MULTIUSER DELAY TRACKING}

\subsection{Delay-robust SIC}

In practical systems, the time delay is not known and must be estimated at the receiver. Denote the estimated time delay of the $l$ th path of the $k$ th user as $\hat{\tau}_{k, l}=\left(\hat{p}_{k, l}+\hat{\delta}_{k, l}\right) T_{c}$, where $\hat{p}_{k, l} \in\{0,1, \ldots, N-1\}$ and $\hat{\delta}_{k, l} \in[0,1)$ is the fractional part.

Since most current time delay estimation methods have a delay estimation error standard deviation within $0.1 T_{c}[5$, 6], we assume that the delay error $\Delta \tau_{k, l}=\tau_{k, l}-\hat{\tau}_{k, l}$ is small compared to $T_{c}$.

The effect of imperfect time delay estimation, that is, delay mismatch, on the performance of multiuser detectors has been investigated in [4]. It is shown that these multiuser detectors are all sensitive to delay mismatch; BER performance degrades greatly even with relatively small delay estimation errors under severe near-far scenarios.

Modified multiuser detectors have thus been proposed to mitigate the effects of imperfect time delay estimation. The deterministic approach [17] applies only to rectangular chippulse shapes and has a capacity limit of $50 \%$ of the spreading factor. The stochastic approaches $[18,19]$ depend on the delay error distribution. The residual MAI caused by timing error is not completely eliminated and stochastic approaches are, therefore, not near-far resistant.

We adopt a delay-robust multiuser receiver based on SIC implementation which is denoted as delay-robust SIC [20]. At each SIC iteration, the timing delay error introduced interference is estimated and cancelled.

When the delay error is small, the $k$ th user's signature waveform for the $i$ th interval and the $l$ th path, $\mathbf{d}_{k, l}(i)$, is firstorder Taylor expanded, as shown in Figure 1,

$$
\mathbf{d}_{k, l}(i) \approx \hat{\mathbf{d}}_{k, l}(i)+\left(\delta_{k, l}-\hat{\delta}_{k, l}\right) \Delta \mathbf{d}_{k, l}(i),
$$

where $\hat{\mathbf{d}}_{k, l}(i)$ is similar to $\mathbf{d}_{k, l}(i)$ with $\hat{\tau}_{k, l}$ replacing $\tau_{k, l}$. 


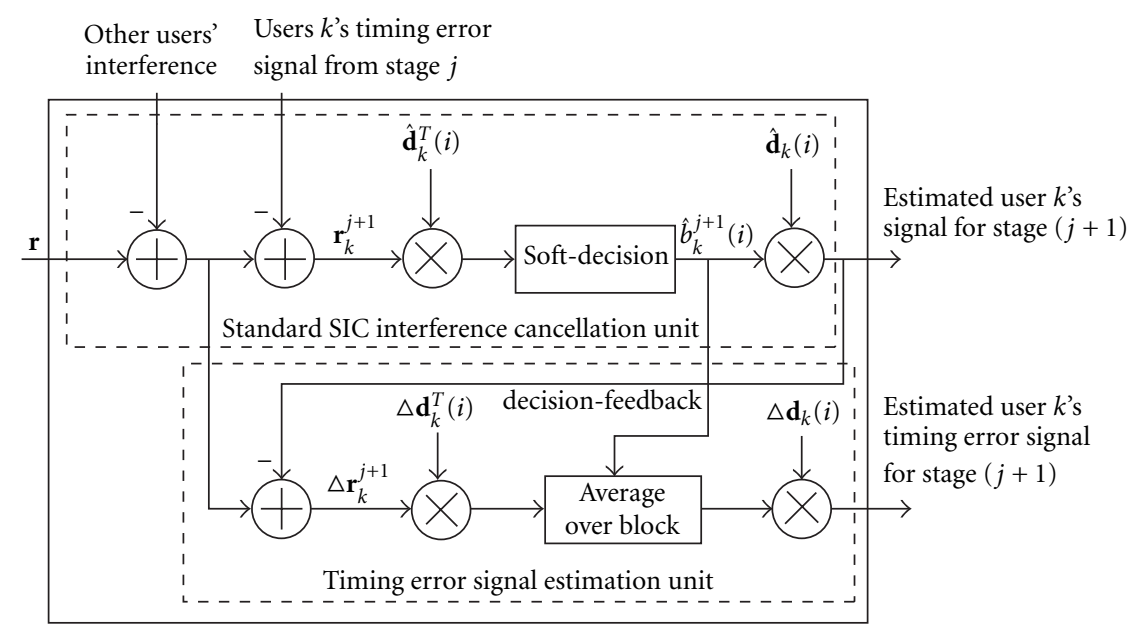

FIGURE 2: Interference cancellation unit of delay-robust SIC for user $k$ at the $(j+1)$ st stage.

The error vector $\Delta \mathbf{d}_{k, l}(i)$ for the band-limited chip pulse shape is the convolution of the user spreading codes with the first derivative vector $\mathrm{f}_{k, l}$ of the $l$ th path of the $k$ th user's chipmatched filter response at the sampling points, that is,

$$
\Delta \mathbf{d}_{k, l}(i)=\mathbf{c}_{k}\left(p_{k, l}, i\right) * \mathbf{f}_{k, l}
$$

with elements

$$
\begin{gathered}
f_{k, l}(m)=\left.\frac{\partial}{\partial t} \int_{-\infty}^{\infty} h\left(\tau-\delta_{k, l} T_{c}\right) h^{*}(t-\tau) d \tau\right|_{t=m T_{c}} \\
m \in\{1,2, \ldots, P\} .
\end{gathered}
$$

In the special case of a rectangular chip pulse, $P=2$ and $\mathbf{f}_{k, l}=\left[\begin{array}{ll}-1 & 1\end{array}\right]$, the error vector

$$
\Delta \mathbf{d}_{k, l}(i)=-\mathbf{c}_{k}\left(\hat{p}_{k, l}, i\right)+\mathbf{c}_{k}\left(\hat{p}_{k, l}+1, i\right) .
$$

If rectangular chip pulses are used, the expansion in (11) is exact [19]. We note that the linear approximation in (11) for band-limited chip pulses is a good approximation in tracking mode where the timing error is small.

The performance degradation of the CDMA multiuser detectors is caused by the delay error signal which is a multiple of the error vector $\Delta \mathbf{d}_{k, l}(i)$. Since the error vector $\Delta \mathbf{d}_{k, l}(i)$ is known, we may estimate the amplitude of error signal by using the tentative decision-feedback in the iterations of the SIC and cancel this interference. Robustness to time delay error is achieved by this improved SIC algorithm under nearfar conditions.

To improve the estimation accuracy of the error signal at each iteration, the $M$ error vectors of each user are combined into a long error vector, $\mathbf{e}_{k, l}$, based on the tentative data symbol decisions, $\hat{b}_{k}(i)$. If the complex fading gains $\alpha_{k, l}(i)$ are known, then

$$
\mathbf{e}_{k, l}=\frac{1}{M} \sum_{i=1}^{M} \Delta \mathbf{d}_{k, l}(i) \hat{b}_{k}(i) \alpha_{k, l}(i)
$$

We note that this is actually averaging the estimate over a window of $M$ symbols.
To simplify the algorithm description, we let the number of paths be $L=1$ and drop the subscript $l$ for the paths. The delay-robust SIC algorithm is as follows [21], where its interference cancellation unit for the $k$ th user at stage $(j+1)$ is as shown in Figure 2.

\section{Initialization:}

Calculate all $\hat{\mathbf{d}}_{k}(i)$ and $\Delta \mathbf{d}_{k}(i)$, and set all initial values to zeros.

Iteration:

For $j=1,2, \ldots, J$ do:

For $k=1,2, \ldots, K$ do:

Steps (1) through (4)

(1) estimate user $k$ 's received signal for the $(j+$ 1)st iteration by subtracting other users' reconstructed signals and error signals from the received signal

$$
\begin{aligned}
\mathbf{r}_{k}^{j+1}= & \mathbf{r}-\sum_{l=1}^{k-1}\left(\hat{\mathbf{r}}_{l}^{j+1}+\widehat{\Delta a}_{l}^{j+1} \mathbf{e}_{l}^{j+1}\right) \\
& -\sum_{l=k+1}^{K}\left(\hat{\mathbf{r}}_{l}^{j}+\widehat{\Delta a}_{l}^{j} \mathbf{e}_{l}^{j}\right)-\widehat{\Delta a}_{k}^{j} \mathbf{e}_{k}^{j},
\end{aligned}
$$

where

$$
\begin{aligned}
\mathbf{e}_{l}^{j} & =\sum_{i=1}^{M} \Delta \mathbf{d}_{l}(i) \hat{b}_{l}^{j}(i) \alpha_{l}(i), \\
\hat{\mathbf{r}}_{l}^{j} & =\sum_{i=1}^{M} \hat{b}_{l}^{j}(i) \alpha_{l}(i) \hat{\mathbf{d}}_{l}(i) \quad \text { for } l \geq k, \\
\mathbf{e}_{l}^{j+1} & =\sum_{i=1}^{M} \Delta \mathbf{d}_{l}(i) \hat{b}_{l}^{j+1}(i) \alpha_{l}(i), \\
\hat{b}_{l}^{j+1}(i) & =f_{\operatorname{dec}}\left(\tilde{b}_{l}^{j+1}(i)\right), \\
\hat{\mathbf{r}}_{l}^{j+1} & =\sum_{i=1}^{M} \hat{b}_{l}^{j+1}(i) \alpha_{l}(i) \hat{\mathbf{d}}_{l}(i) \quad \text { for } l<k .
\end{aligned}
$$


(2) For each symbol in the block, $i=1, \ldots, M$, obtain the normalized soft data symbol estimate and make a data symbol decision. For the $i$ th symbol, the soft data symbol estimate is normalized with respect to $\alpha_{k}(i)$,

$$
\tilde{b}_{k}^{j+1}(i)=\operatorname{Re}\left(\frac{\left(\hat{\mathbf{d}}_{k}(i)\right)^{T} \mathbf{r}_{k}^{j+1}}{\alpha_{k}(i)}\right),
$$

where $\operatorname{Re}(\cdot)$ takes the real value. The data symbol decision is then made by the decision function $f_{\mathrm{dec}}(\cdot)$,

$$
\hat{b}_{k}^{j+1}(i)=f_{\operatorname{dec}}\left(\tilde{b}_{k}^{j+1}(i)\right) .
$$

(3) Estimate the error signal of the $k$ th user due to time delay error as

$$
\Delta \mathbf{r}_{k}^{j+1}=\mathbf{r}_{k}^{j+1}-\hat{\mathbf{r}}_{k}^{j+1}+\widehat{\Delta a}_{k}^{j} \mathbf{e}_{k}^{j} .
$$

(4) Update the normalized amplitude of the error signal,

$$
\begin{aligned}
\mathbf{e}_{k}^{j+1} & =\sum_{i=1}^{M} \Delta \mathbf{d}_{k}(i) \hat{b}_{k}^{j+1}(i) \alpha_{k}(i), \\
\widehat{\Delta a}_{k}^{j+1} & =\left(\mathbf{e}_{k}^{j+1}\right)^{H}\left(\Delta \mathbf{r}_{k}^{j+1}\right) .
\end{aligned}
$$

We note that since $\widehat{\Delta a}_{k}=\left(\delta_{k}-\hat{\delta}_{k}\right)$ from (11), the estimate of the normalized error signal amplitude $\widehat{\Delta a}$ at $(j+1)$ st iteration in (22) can be used to improve the delay estimate.

\subsection{Delay tracking for known fading channels}

We propose using a sliding window version of the delayrobust SIC as a multiuser delay-tracking receiver. The values computed during the previous window can be used as initial values for the current window computation so the computation complexity can be greatly reduced. It is required that all users are initially under acquisition, that is, within $\pm 0.5 T_{c}$ of the true delay. This initial acquisition can be obtained by using other delay estimation algorithms $[5,6]$.

The delay is updated in small steps, that is, $0.05 T_{c}$. When the delay error estimate is larger than $\pm 0.05 T_{c}$, the user time delay is updated by $\pm 0.05 T_{c}$ and then used in the next window where the same procedure is repeated.

We note that there are implementation advantages when the time delays are updated in fixed small steps; the recalculation of the user signature sequence according to (11) is avoided if the delay change is small. Sudden large changes, due to impulse noise or interference, are also avoided.

It was shown in [12] that when the observation window length $M$ is larger than 8 symbols, edge effects can be neglected. It is also shown in [20] that the delay-robust receiver has a capacity of $M /(M+1)$ of the standard SIC. As a compromise, we choose $M=9$ in our simulations.

The delay-robust SIC adds an error signal estimation procedure to the standard multistage SIC. As pointed in [20], this delay-robust SIC implicitly incorporates soft error signal cancellation into its iterations; when some symbols of the tentative data symbol decisions are incorrect, the estimated amplitude of the error signal will be smaller than the actual value, which is equivalent to soft cancellation with a factor less than $100 \%$. Thus, this delay-robust SIC is numerically stable.

When the complex fading channel gains $\alpha_{k, l}(i)$ are known, which is the case of slow fading or additive white Gaussian noise (AWGN) channels where the channel gains and amplitudes can be easily estimated, we may use a softdecision function in the SIC to improve BER performance. One possible soft-decision function that leads to good performance is [21]

$$
\hat{b}=f_{\operatorname{dec}}(\tilde{b})= \begin{cases}1, & \tilde{b}>c, \\ \tilde{b}, & \tilde{b} \in[-c, c], \\ -1, & \tilde{b}<-c,\end{cases}
$$

where the threshold $c$ satisfies $0.0 \leq c \leq 1.0$. The effect of the choice of $c$ on the performance of the SIC using the above soft-decision function was analyzed and simulated in [21]. It was found that $c=0.5$ is a good choice, so we use $c=0.5$ throughout this paper if soft decision is applied.

\subsection{Delay tracking for unknown fading channels}

Channel estimation for a fast fading multiuser CDMA channel is a difficult problem, since the effect of MAI must be suppressed or eliminated first to obtain an accurate channel estimate for the desired user. One suboptimum solution is the multipath decorrelating detector.

The multipath decorrelating detector can be first used to decorrelate each path of each user before channel estimation. The channel estimator works on these MAI-free decorrelator outputs [22]. The price paid for this MAI-free decorrelation is the noise enhancement and reduced system capacity since each user is detected as $L$ users.

As shown in [23], the linear SIC (multistage SIC with linear-decision function) is an iterative implementation of the decorrelator. The linear-decision function is $f_{\mathrm{dec}}(x)=x$ which is equivalent to estimate the data symbol $b_{k}(i)$ and complex channel gain $\alpha_{k, l}(i)$ as a composite signal $o_{k, l}(i)$ at stage $(j+1)$,

$$
\hat{o}_{k, l}^{j+1}(i)=\left(\mathbf{d}_{k, l}(i)\right)^{T} \mathbf{r}_{k, l}^{j+1},
$$

and $\mathbf{e}_{k, l}$ at stage $(j+1)$ is

$$
\mathbf{e}_{k, l}^{j+1}=\sum_{i=1}^{M} \Delta \mathbf{d}_{k, l}(i) \hat{o}_{k, l}^{j+1}(i) .
$$

With this new $\mathbf{e}_{k, l}^{j+1}$, we replace $\hat{b}_{k}^{j}(i) \alpha_{k}(i)$ by $\hat{o}_{k}^{j}(i)$ in Step (1) and we replace the entire Step (2) by (24). The resulting delay-robust SIC with a linear-decision function can be used to track the time delays of all users for fast fading channels.

\subsection{Implementation issues}

SIC is a low complexity suboptimum CDMA multiuser detection method and has been considered to be practical for 
actual CDMA basestation implementation [24]. It can be shown from Figure 2 that through the added timing error estimation and cancellation, the proposed delay-robust SIC approximately doubles both computational complexity and processing delay, compared to the standard SIC algorithm [2]. As a function of the number of users, the complexity and processing delay are of the same order of magnitude as the standard SIC.

\section{NUMERICAL AND SIMULATION RESULTS}

In this section, we will first evaluate the BER performance of the delay-robust SIC for a static channel using PN chiplevel simulation. The sliding window version of the delayrobust SIC is then used to track the multiple user time delay variations for both the rectangular chip pulses and the bandlimited chip pulses.

Throughout the simulations, Gold code sequences of length $N=31$ are used. The number of users is fixed at $K=20$ to account for a highly loaded system. The signalto-noise ratio (SNR) is defined with respect to the user of interest, user 1 . The near-far ratio is defined as the power ratio between the strongest user and user 1 which is fixed at $10 \mathrm{~dB}$. All other users have an amplitude uniformly distributed between those of the strongest user and the weakest user.

First, we consider the AWGN channel. Here, we can take advantage of the constant amplitude property by using a softdecision function. The amplitude is estimated by averaging over a block of $M$ to reduce its noise variance by a factor of $M$ [2]. The threshold used for the soft-decision function is $c=0.5$, and the total number of interference cancellation stages is $J=5$. The tracking for fast fading channels using a linear-decision function is simulated inSection 4.4 with $J=$ 10 interference cancellation stages.

\subsection{BER performance}

For the simulation of BER, a window size of $M=9$ is used. Rectangular chip pulses are used. The estimated time delay is static, that is, fixed over the observation window with a delay error standard deviation of $\sigma_{\tau}=0.1 T_{c}$.

In Figure 3, the BER curve of the delay-robust SIC with soft-decision and amplitude averaging is shown as a function of SNR. As a comparison, the BER curve of the single user case is shown as a lower bound which can only achieved by the ideal multiuser detector with true time delay and amplitude information. From Figure 3, the delay-robust SIC performance is only about $1.2 \mathrm{~dB}$ away from the single user bound at a BER of $10^{-3}$. The BER curves of the decorrelating detector with both true time delay and estimated time delays are also shown. Both of their performances are far from the single user bound because of the decorrelator's noise enhancement.

In Figure 4, we compare the BER performance of the delay-robust SIC and the standard SIC detectors [2] under two different delay error conditions of $\sigma_{\tau}=0.1 T_{c}$ and $\sigma_{\tau}=0.2 T_{c}$, respectively. As shown, the delay-robust SIC is much more insensitive to the $\sigma_{\tau}$ increase. This indicates that

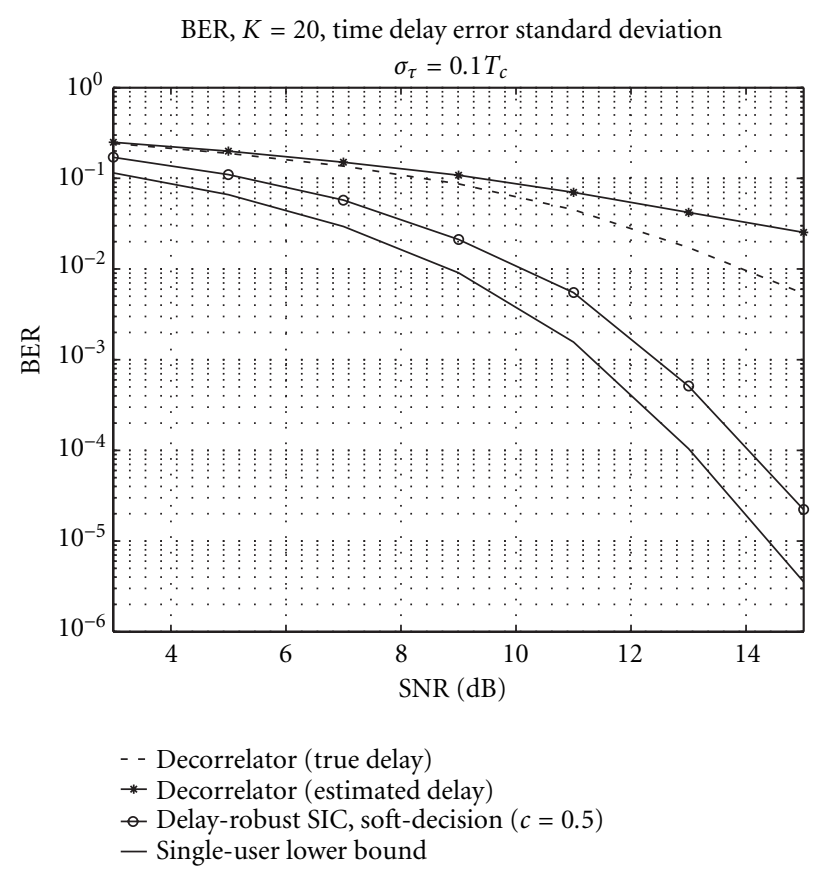

Figure 3: Bit error rate (BER) of user 1 for delay-robust SIC detector. Near-far ratio $=10 \mathrm{~dB}$. Soft-decision function is used with threshold $c=0.5$.

it is advantageous to jointly estimate delay errors and detect data symbols.

\subsection{Tracking for rectangular chip pulses}

For simulating the tracking of multiuser time delays, data symbols are assumed to be transmitted continuously. We let sliding window length be $M=9$ symbols.

In this simulation, rectangular chip pulses are used. We tracked a total of $K=20$ users' delays. The SNR of the weakest user is $14 \mathrm{~dB}$. The weakest user's and the strongest user's delay tracking curves are shown in Figure 5 where the solid line and the dashed line represent the actual and estimated delay trajectories, respectively. The initial acquisition has a delay error standard deviation of $0.5 T_{c}$. The spreading factor is $N=31$. The update step of the delay tracker is $0.05 T_{c}$.

We assume the delay varies with time as a first-order Gauss-Markov process as in [7]

$$
\tau(m+1)=\tau(m)+w(m)+u(m),
$$

where $w(m)$ is a zero-mean noise process with variance $\sigma_{w}^{2}$ and $u(m)$ is a deterministic scalar that models slow global drift. We use $\sigma_{w}^{2}=0.01 \sigma^{2}$. The global time variation $u(m)$ is selected to change at a rate of $0.005 T_{c}$ per symbol. Assuming a propagation speed of $3.0 \times 10^{8} \mathrm{~m} / \mathrm{s}$, this is equivalent to $(0.005 / 31) \times 3.0 \times 10^{8} \approx 5 \times 10^{4} \mathrm{~m} / \mathrm{s}$, or $1.8 \times 10^{5} \mathrm{~km} / \mathrm{h}$ [8].

As we can see, the delay-robust SIC based delay tracker can track the time delays for both the strongest user and the weakest user. In Figure 6, the BER of the delay-robust SIC using dynamic tracking is compared to the BER of the 


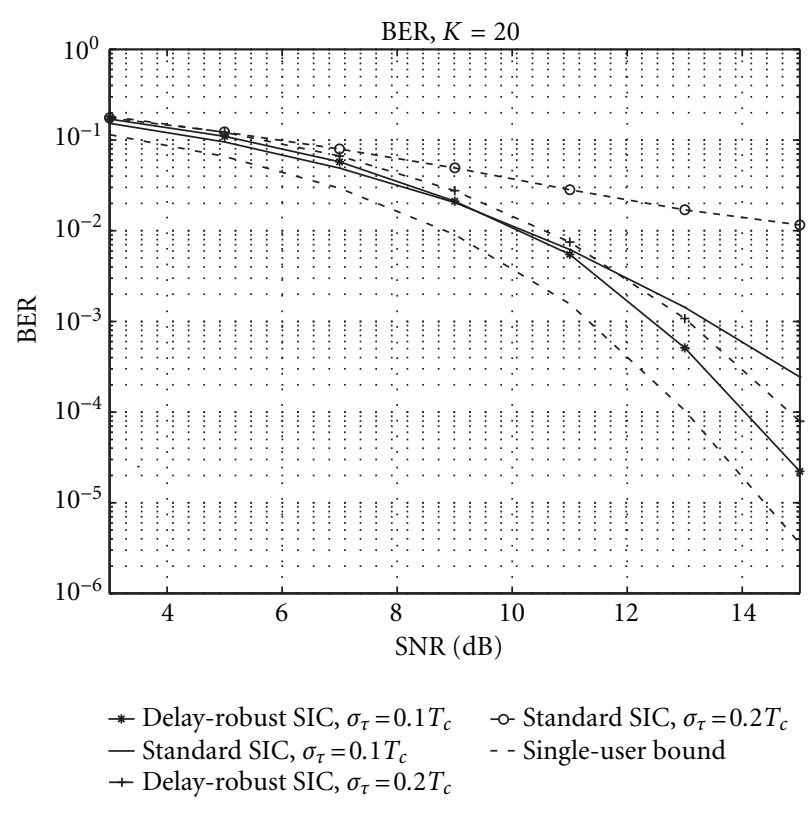

FIgURE 4: BER of user 1 for delay-robust SIC and standard SIC detectors. Near-far ratio $=10 \mathrm{~dB}$. Soft-decision function is used with threshold $c=0.5$.

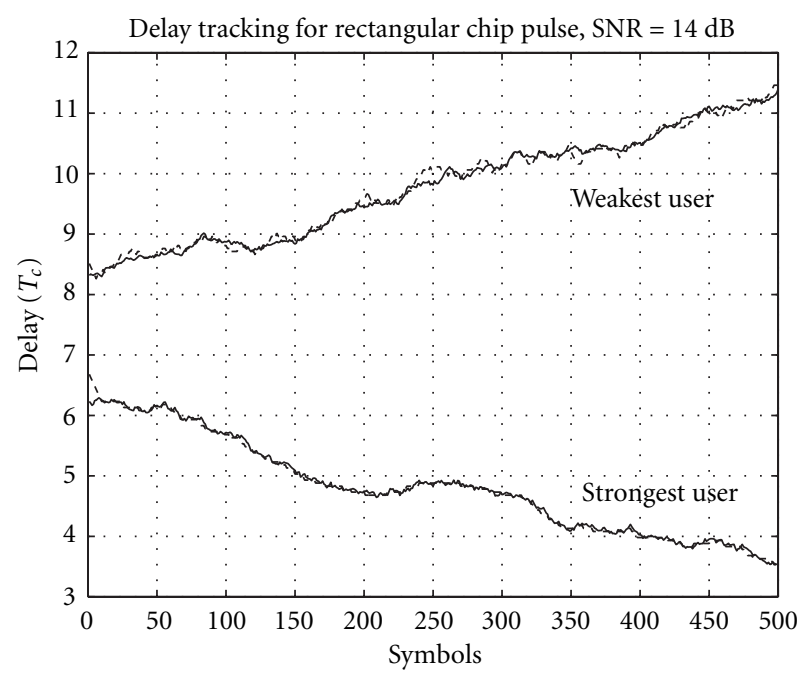

FIGURE 5: Delay tracking curves of delay-robust SIC. Near-far ratio $=10 \mathrm{~dB}$. The weakest user has SNR $=14 \mathrm{~dB}$. Soft-decision function is used with threshold $c=0.5$. The solid line is the true delay and the dashed line is the tracking result.

delay-robust SIC in the static model of Section 4.1. The time delay variation in the tracking model adds only a small amount of noise, and the BER error floor is not obvious.

From Figure 6, the delay tracking multiuser receiver has a BER of approximately $20 \%$ for the weakest user at SNR of $3 \mathrm{~dB}$. Even for such high BER, the delay-robust SIC based delay tracker can still follow the delay variation as shown in Figure 7.

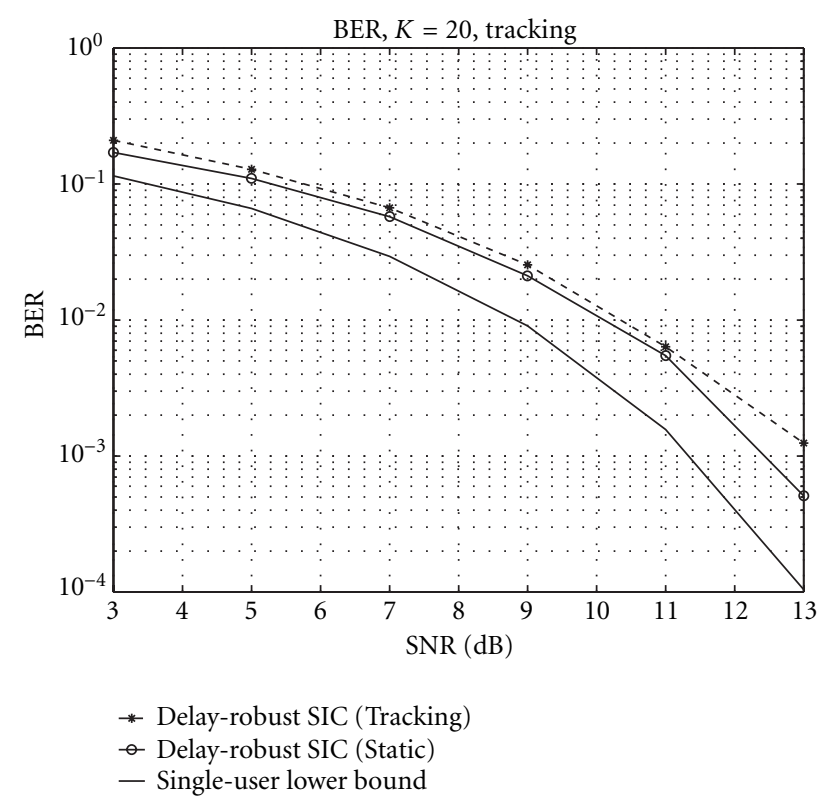

FIGURE 6: BER of user 1 for delay-robust SIC detector in tracking time delays. Near-far ratio $=10 \mathrm{~dB}$. Soft-decision function is used with threshold $c=0.5$.

\subsection{Tracking for band-limited chip pulses}

In Figure 8, a square-root raised cosine chip pulse with rolloff factor 0.35 is used. The SNR of the weakest user is $14 \mathrm{~dB}$. Since band-limited chip pulse is more sensitive to the time delay errors, the initial acquisition delay error standard deviation is reduced to $0.1 T_{c}$, and the update step is smaller, $0.01 T_{c}$. We found that for band-limited chip pulses, the delay tracking algorithm cannot track the time variations of Section 4.2, so we used more mild conditions, that is, we set $\sigma_{w}^{2} 10 \mathrm{~dB}$ lower and reduce the global drift to $0.001 T_{c}$ per symbol.

\subsection{Tracking for time varying fading channels}

In Section 4.2, we have assumed that the complex channel gain remains constant while only the user time delays are varying. However, in wireless mobile channels, the user time delays vary relatively slowly compared to the variation of the complex-channel gains.

The delay-robust SIC with a linear-decision function is used to track the delay variations. Since the data symbol $b_{k}(i)$ and complex channel gain $\alpha_{k, l}(i)$ are estimated as a composite signal $\hat{o}_{k, l}(i)$, data detection is not possible before knowing $\alpha_{k, l}(i)$. The tracking results are shown in Figure 9 for the weakest and strongest users. The SNR of the weakest user is $14 \mathrm{~dB}$. The time variation is the same as in Section 4.2. Timecorrelated Rayleigh fading channel gains are generated with normalized Doppler fading rate of $f_{D} T=0.01$. Because of the noisy estimate $\hat{o}_{k, l}(i)$, the tracking performance of the weakest user is reduced. Note that this simulation is approximately equivalent to a $K=10$ user system with $L=2$ paths for each user. 


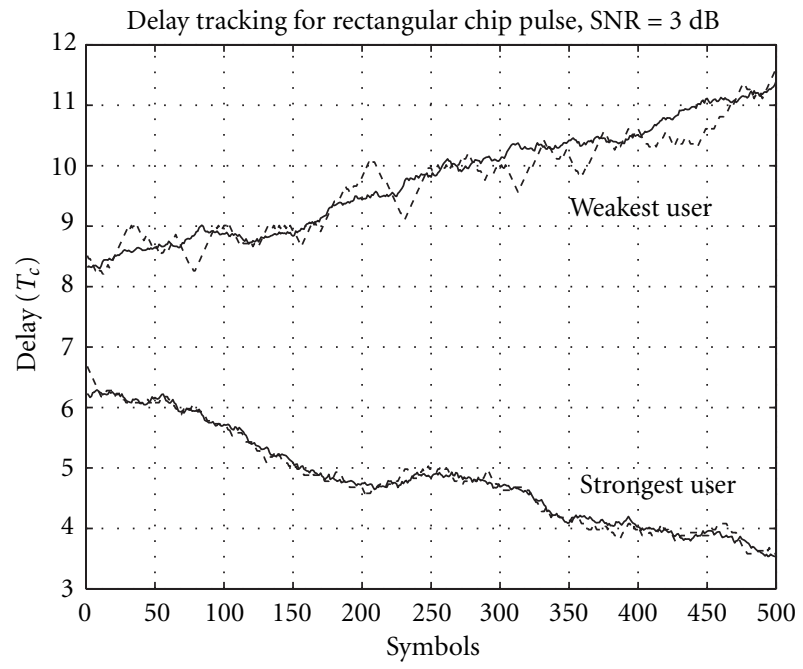

FIgURE 7: Delay tracking curves of delay-robust SIC. Near-far ratio $=10 \mathrm{~dB}$. The weakest user has SNR $=3 \mathrm{~dB}$. Soft-decision function is used with threshold $c=0.5$. The solid line is the true delay and the dashed line is the tracking result.

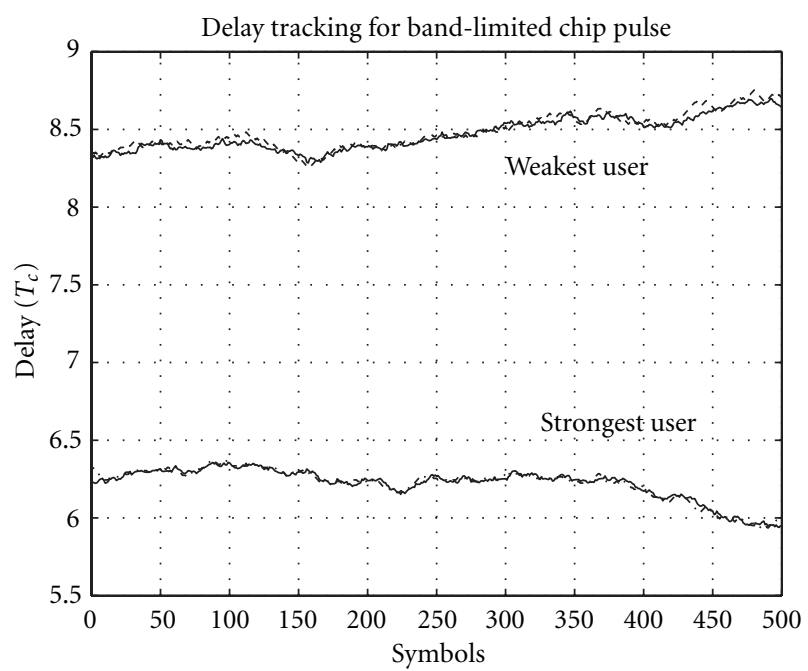

FIgURE 8: Delay tracking curves of delay-robust SIC for bandlimited chip pulses. Near-far ratio $=10 \mathrm{~dB}$. The weakest user has $\mathrm{SNR}=14 \mathrm{~dB}$. Soft-decision function is used with threshold $c=0.5$. The solid line is the true delay and the dashed line is the tracking result.

By assuming the exact knowledge of the transmitted data symbol $b_{k}(i)$, the complex channel gain is tracked in Figure 10 for user 1 . The delay-robust SIC decorrelates the fading signal for different users, so it can be used to track both delay and channel variations.

\section{DISCUSSION}

Although we have demonstrated delay tracking using noisy estimates of the composite signal, it is desirable to have a

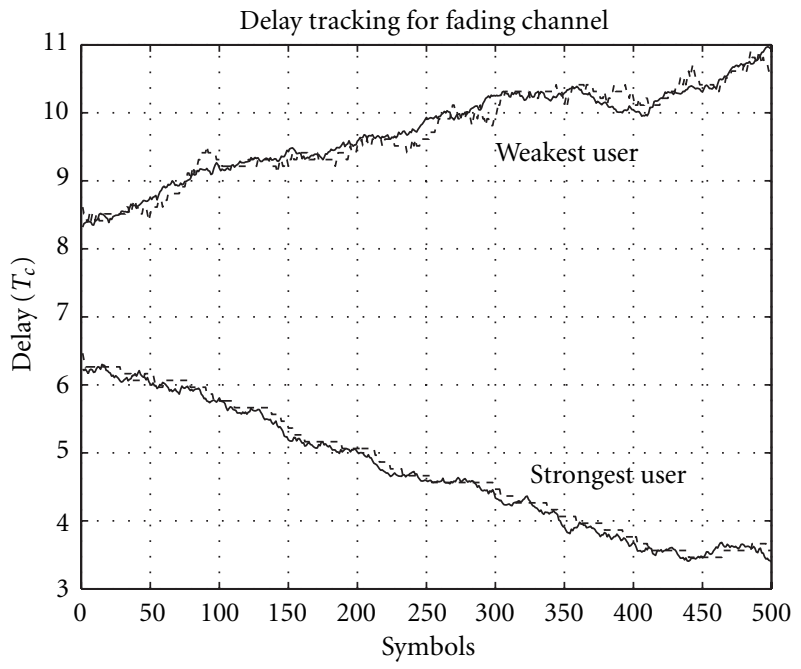

FIGURE 9: Delay tracking curves of delay-robust SIC for Rayleigh fading channels. Normalized Doppler fading rate is $f_{D} T=0.01$. Near-far ratio $=10 \mathrm{~dB}$. The weakest user has SNR $=14 \mathrm{~dB}$. Lineardecision function is used. The solid line is the true delay and the dashed line is the tracking result.
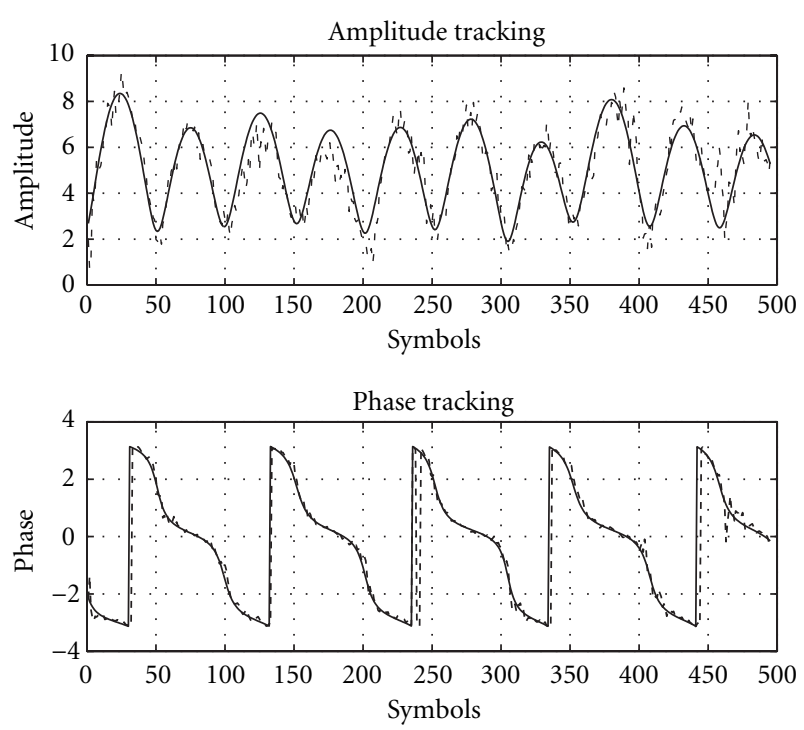

FIGURE 10: Channel amplitude and phase tracking curves of delayrobust SIC for Rayleigh fading channels for the weakest user. Normalized Doppler fading rate is $f_{D} T=0.01$. The weakest user has $\mathrm{SNR}=14 \mathrm{~dB}$. Linear-decision function is used. The solid line is the true delay and the dashed line is the tracking result.

smoothed estimate. In addition, for multipath fading channels, it is advantageous to coherently combine the multipath signals before data detection. Coherent diversity combining requires the channel estimates $\alpha_{k, l}(i)$, not just the composite signal $o_{k, l}(i)[22,25]$.

In [22], the multipath decorrelator is followed by $K L$ adaptive channel estimators using the least mean square (LMS) algorithm assuming the channel fading follows the 
Gauss-Markov model. In [26], disjoint Kalman filtering channel estimation is applied where each user uses a Kalman filter to estimate its channel coefficients based also on the MAI-free decorrelator outputs. However, the use of the multipath decorrelator or multipath linear MMSE receiver as the front-end for the channel estimator can only be applied to the case of relatively few users because of the loss of the degrees of freedom in the signal subspace as the number of users gets large [27].

Multiuser channel estimation that does not use a multipath decorrelator front-end may have less noise enhancement and larger capacity for relatively slow fading where there is no severe tracking problem. In [25], pilot symbols are periodically inserted for channel estimation using a Hamming windowed finite impulse response (FIR) low-pass filter to approximate the optimal Wiener filter. Interpolation is used to obtain the channel estimate for other symbols for the multistage SIC receiver [25]. The performance of a pilot symbol aided channel estimator with interpolation for a single user channel was analyzed in [28]. In [10, 11], a PIC-based channel estimator uses a decision-directed linear predictor and a linear smoother which were previously proposed for single-user channel estimation [29].

As seen in Section 4, the user-delay variation in a practical system is far less than that used in our simulations. In our simulations, we have averaged the time delay error over a block of $M=9$ symbols to filter out the noise. Although the delay-robust SIC based multiuser delay tracker does track the multiuser delay changes, it sometimes loses tracking because of the small block length used. In practice, the delay error can be averaged over a longer block, that is, $M=$ 70 symbols, to have better tracking performance. In practical implementations, the block averaging may be replaced by exponential weighting with a forgetting factor less than unity.

The channel estimation and delay tracking can be separated by using different block lengths for prediction or smoothing by using our delay-robust SIC technique, similar to the PIC-based delay tracker $[10,11]$. This provides an alternative to the EKF based channel and delay trackers $[7,8,9]$. The EKF was introduced since the time delay is a nonlinear function of the user signature sequence. When channel and delay estimation are separated, the simpler Kalman filter can be used for the channel estimation, while the delay tracking can be handled by the delay-robust SIC using a longer smoothing length.

\section{CONCLUSION}

We have proposed to use a delay-robust SIC detector for multiuser time delay tracking. The time delay error vector in the robust SIC can be used to estimate the time delay changes of all users. The delay-robust SIC implicitly combined the delay tracking into the multiuser detection. For a static delay channel, the delay-robust SIC has a BER close to the single-user bound. For dynamic delay tracking, the BER of the multiuser delay-tracking receiver, based on the sliding window implementation of delay-robust SIC, is only slightly inferior to that of the static channel. Accurate tracking occurs for both rectangular and bandlimited chip pulses and for both constant and fast fading channels.

\section{ACKNOWLEDGMENT}

This research has been supported by the Canadian Institute for Telecommunications Research under the NCE program of the Government of Canada.

\section{REFERENCES}

[1] S. Verdú, "Minimum probability of error for asynchronous Gaussian multiple-access channels," IEEE Transactions on Information Theory, vol. 32, no. 1, pp. 85-96, 1986.

[2] P. Patel and J. Holtzman, "Analysis of a simple successive interference cancellation scheme in a DS/CDMA system," IEEE Journal on Selected Areas in Communications, vol. 12, no. 5, pp. 796-807, 1994.

[3] D. Koulakiotis and A. H. Aghvami, "Data detection techniques for DS/CDMA mobile systems: a review," IEEE Personal Communications, vol. 7, no. 3, pp. 24-34, 2000.

[4] S. Parkvall, E. G. Ström, and B. Ottersten, "The impact of timing errors on the performance of linear DS-CDMA receivers," IEEE Journal on Selected Areas in Communications, vol. 14, no. 8, pp. 1660-1668, 1996.

[5] E. G. Ström, S. Parkvall, S. L. Miller, and B. Ottersten, "Propagation delay estimationin asynchronous direct-sequence code-division multiple access systems," IEEE Trans. Communications, vol. 44, no. 1, pp. 84-93, 1996.

[6] S. E. Bensley and B. Aazhang, "Subspace-based channel estimation for code division multiple access communication systems," IEEE Trans. Communications, vol. 44, no. 8, pp. 10091020, 1996.

[7] T. J. Lim and L. K. Rasmussen, "Adaptive symbol and parameter estimation in asynchronous multiuser CDMA detectors," IEEE Trans. Communications, vol. 45, no. 2, pp. 213220, 1997.

[8] J. Caffery Jr. and G. L. Stüber, "Nonlinear multiuser parameter estimation and tracking in CDMA systems," IEEE Trans. Communications, vol. 48, no. 12, pp. 2053-2063, 2000.

[9] R. A. Iltis, "A DS-CDMA tracking mode receiver with joint channel/delay estimation and MMSE detection," IEEE Trans. Communications, vol. 49, no. 10, pp. 1770-1779, 2001.

[10] M. Latva-aho and J. Lilleberg, "Parallel interference cancellation based delay tracker for CDMA receivers," in Proc. the 30th Conference on Information Sciences and Systems (CISS96), pp. 852-857, Princeton, NJ, USA, March 1996.

[11] M. Latva-aho and J. Lilleberg, "Delay trackers for multiuser receivers," in Proc. Int. Conf. Universal Personal Communications, pp. 326-330, Boston, Mass, USA, September 1996.

[12] M. J. Juntti and B. Aazhang, "Finite memory-length linear multiuser detection for asynchronous CDMA communications," IEEE Trans. Communications, vol. 45, no. 5, pp. 611622, 1997.

[13] J. Shen and Z. Ding, "Edge decision assisted decorrelators for asynchronous CDMA channels," IEEE Trans. Communications, vol. 47, no. 3, pp. 438-445, 1999.

[14] J. G. Proakis, Digital Communications, WCB/McGraw-Hill, Boston, Mass, USA, 3rd edition, 1995. 
[15] P. Xiao and E. G. Ström, "Synchronization algorithms for iterative demodulated M-ary DS-CDMA systems," in Proc. IEEE Global Telecommunications Conference, pp. 1371-1375, San Antonio, Tex, USA, November 2001.

[16] M. Guenach and L. Vandendorpe, "Design and performance analysis of DA multiuser path trackers for long code DSCDMA systems," in Proc. IEEE Global Telecommunications Conference, vol. 2, pp. 1381-1385, San Antonio, Tex, USA, November 2001.

[17] F. van Heeswyk, D. D. Falconer, and A. U. H. Sheikh, "A delay independent decorrelating detector for quasi-synchronous CDMA," IEEE Journal on Selected Areas in Communications, vol. 14, no. 8, pp. 1619-1626, 1996.

[18] L.-C. Chu and U. Mitra, "Performance analysis of an improved MMSE multi-user receiver for mismatched delay channels," IEEE Trans. Communications, vol. 46, no. 10, pp. 1369-1380, 1998.

[19] T. Östman, M. Kristensson, and B. Ottersten, "Asynchronous DS-CDMA detectors robust to timing errors," in Proc. IEEE Vehicular Technology Conference, vol. 3, pp. 1704-1708, Phoenix, Ariz, USA, January 1997.

[20] W. Zha and S. D. Blostein, "Improved CDMA multiuser receivers robust to time errors," in Proc. IEEE Int. Conf. Acoustics, Speech, Signal Processing, Salt Lake City, Utah, USA, May 2001.

[21] W. Zha and S. D. Blostein, "Soft-decision successive interference cancellation CDMA receiver with amplitude averaging and robust to timing errors," in Proc. IEEE Global Telecommunications Conference, San Antonio, Tex, USA, November 2001.

[22] M. Stojanovic and Z. Zvonar, "Performance of multiuser detection with adaptive channel estimation," IEEE Trans. Communications, vol. 47, no. 8, pp. 1129-1132, 1999.

[23] L. K. Rasmussen, T. J. Lim, and A. L. Johansson, "A matrixalgebraic approach to successive interference cancellation in CDMA," IEEE Trans. Communications, vol. 48, no. 1, pp. 145$151,2000$.

[24] I. Seskar and N.B. Mandayam, "Software-defined radio architectures for interference cancellation in DS-CDMA systems," IEEE Personal Communications, vol. 6, no. 4, pp. 26-34, 1999.

[25] A. C. K. Soong and W. A. Krzymien, "A novel CDMA multiuser interference cancellation receiver with reference symbol aided estimation of channel parameters," IEEE Journal on Selected Areas in Communications, vol. 14, no. 8, pp. 1536-1547, 1996.

[26] P. H. Wu and A. Duel-Hallen, "Multiuser detectors with disjoint Kalman channel estimators for synchronous CDMA mobile radio channels," IEEE Trans. Communications, vol. 48, no. 5, pp. 752-756, 2000.

[27] M. Latva-Aho and M. J. Juntti, "LMMSE detection for DSCDMA systems in fading channels," IEEE Trans. Communications, vol. 48, no. 2, pp. 194-199, 2000.

[28] J. K. Cavers, "An analysis of pilot symbol assisted modulation for Rayleigh fading channels," IEEE Trans. Vehicular Technology, vol. 40, no. 4, pp. 686-693, 1991.

[29] Y. Liu and S. D. Blostein, "Identification of frequency nonselective fading channels using decision feedback and adaptive linear prediction," IEEE Trans. Communications, vol. 43, no. 2/3/4, pp. 1484-1492, 2-4 1995.
Wei Zha received his B.S. and M.S. from Shanghai Jiao Tong University, Shanghai, China, in 1992 and 1995, respectively, both in electronics engineering. He worked as a Lecturer and Research Engineer at the Department of Electronics Engineering, Shanghai Jiao Tong University, Shanghai, China, from 1995 to 1998 . Since 1998, he has been a Ph.D. student and Research Assistant at the Department of Electrical and

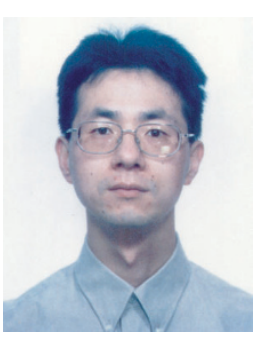
Computer Engineering, Queen's University, Kingston, Canada. His research areas are in CDMA, OFDM, MIMO systems, and general areas of wireless communications and signal processing.

Steven D. Blostein received his B.S. degree in electrical engineering from Cornell University, Ithaca, NY, USA, in 1983, and the M.S. and Ph.D. degrees in electrical and computer engineering from the University of Illinois, Urbana-Champaign, in 1985 and 1988, respectively. He has been on the Faculty at Queen's University since 1988 and currently holds the position of Professor at the Department of Electrical and Computer

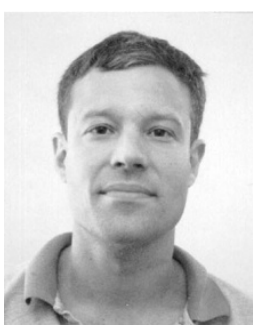
Engineering. He has been a consultant to both industry and government in the areas of document image compression, motion estimation, and target tracking, and was a visiting Associate Professor at the Department of Electrical Engineering at McGill University in 1995. His current interests lie in statistical signal processing, wireless communications, and video image communications. He served as Chair of the IEEE Kingston Section in 1993-94, and Associate Editor for the IEEE Transactions on Image Processing from 19962000. He currently leads the Multirate Wireless Data Access Major Project sponsored by the Canadian Institute for Telecommunications Research. He is a registered Professional Engineer in Ontario. 